\title{
A PLANET IN A CIRCULAR ORBIT WITH A 6 YEAR PERIOD ${ }^{1}$
}

\author{
Brad D. Carter, ${ }^{2}$ R. Paul Butler, ${ }^{3}$ C. G. Tinney, ${ }^{4}$ Hugh R. A. Jones, ${ }^{5}$ Geoffrey W. Marcy, ${ }^{6}$ \\ Chris McCarthy, ${ }^{3}$ Debra A. Fischer, ${ }^{6}$ and Alan J. Penny ${ }^{7}$ \\ Received 2003 June 5; accepted 2003 July 3; published 2003 July 16
}

\begin{abstract}
Precision Doppler velocity measurements from the $3.9 \mathrm{~m}$ Anglo-Australian Telescope reveal a planet with a $6 \mathrm{yr}$ period orbiting the G5 dwarf HD 70642. The $a=3.3$ AU orbit has a low eccentricity $(e=0.1)$, and the minimum $(M \sin i)$ mass of the planet is $2.0 M_{\mathrm{JUP}}$. The host star is metal-rich relative to the Sun, similar to most stars with known planets. The distant and approximately circular orbit of this planet makes it a member of the rarest group to emerge from precision Doppler surveys.
\end{abstract}

Subject headings: planetary systems — stars: individual (HD 70642)

\section{INTRODUCTION}

Of the 77 extrasolar planets currently listed by the IAU Working Group on Extrasolar Planets ${ }^{8}$ (including planet candidates published in refereed journals with $M \sin i<10 M_{\mathrm{JUP}}$ ), only three systems have been found to harbor planets in circular orbits $(e<0.1)$ beyond 0.5 AU: 47 UMa (Fischer et al. 2002; Butler \& Marcy 1996), HD 27442 (Butler et al. 2001), and HD 4208 (Vogt et al. 2002). With 13 "51 Peg-type" planets $(P<5$ days $)$ and $\sim 60$ eccentric planets $(e>0.1)$, the longperiod circular orbits are the rarest of the three types of planetary systems to emerge over the last 8 years.

With one exception, all the IAU Working Group planets orbit within $4 \mathrm{AU}$ of their host stars. Since all these planets have been discovered via the precision Doppler technique, there is a strong selection bias toward discovering systems with small semimajor axes. Unsurprisingly, the only extrasolar planet so far found to orbit beyond $4 \mathrm{AU}$ was detected by the precision Doppler survey that has been gathering data the longest (Marcy et al. 2002).

Perhaps the most critical question facing the field of extrasolar planetary science is whether solar system analogs (i.e., systems with giants planets in circular orbits beyond $4 \mathrm{AU}$ and small rocky planets orbiting in the inner few AU) are ubiquitous or rare. Existing precision Doppler surveys will become sensitive to giant planets orbiting beyond $4 \mathrm{AU}$ by the end of this decade, although only those programs with a long-term precision of $3 \mathrm{~m} \mathrm{~s}^{-1}$ or better will be able to determine whether the orbits of such planets are eccentric or circular (Butler et al. 2001, Fig. 11).

We report here a new extrasolar planet in an approximately

\footnotetext{
${ }^{1}$ Based on observations obtained at the Anglo-Australian Telescope, Siding Spring, Australia.

${ }^{2}$ Faculty of Sciences, University of Southern Queensland, Toowoomba, QLD 4350, Australia; carterb@usq.edu.au.

${ }^{3}$ Department of Terrestrial Magnetism, Carnegie Institution of Washington, 5241 Broad Branch Road, NW, Washington, DC 20015-1305.

${ }^{4}$ Anglo-Australian Observatory, P.O. Box 296, Epping, NSW 1710, Australia.

${ }^{5}$ Astrophysics Research Institute, Liverpool John Moores University, Twelve Quays House, Egerton Wharf, Birkenhead, Wirral, Cheshire CH41 1LD, UK.

${ }^{6}$ Department of Astronomy, University of California at Berkeley, 601 Campbell Hall, Berkeley, CA 94720-3411; and at Department of Physics and Astronomy, San Francisco State University, 1600 Holloway Avenue, San Francisco, CA 94132.

${ }^{7}$ Rutherford Appleton Laboratory, Chilton, Didcot, Oxford OX11 0QX, UK.

${ }^{8} \mathrm{See} \mathrm{http://www.ciw.edu/boss/IAU/div3/wgesp/planets.shtml.}$
}

circular orbit beyond $3 \mathrm{AU}$, discovered with the $3.9 \mathrm{~m}$ AngloAustralian Telescope (AAT). The Anglo-Australian Planet Search program is described in $\S 2$. The characteristics of the host star and the precision Doppler measurements are presented in $\S 3$. A discussion follows.

\section{THE ANGLO-AUSTRALIAN PLANET SEARCH}

The Anglo-Australian Planet Search began in 1998 January and is currently surveying 250 stars. Fourteen planet candidates with $M \sin i$ ranging from $0.2 M_{\mathrm{JUP}}$ to $10 M_{\mathrm{JUP}}$ have first been published with AAT data (Tinney et al. 2001; Butler et al. 2001; Tinney et al. 2002a; Jones et al. 2002a, 2002b; Butler et al. 2002; Tinney et al. 2003; Jones et al. 2003), and an additional four planet candidates have been confirmed with AAT data (Butler et al. 2001).

Precision Doppler measurements are made with the University College London Echelle Spectrograph (UCLES; Diego et al. 1990). An iodine absorption cell (Marcy \& Butler 1992) provides the wavelength calibration from 5000 to $6000 \AA$. The spectrograph point-spread function and wavelength calibration are derived from the embedded iodine lines (Valenti, Butler, \& Marcy 1995; Butler et al. 1996). This system has demonstrated a long-term precision of $3 \mathrm{~m} \mathrm{~s}^{-1}$ (Butler et al. 2001), similar to (if not exceeding) the iodine systems on the Lick $3 \mathrm{~m}$ telescope (Butler et al. 1996; Butler \& Marcy 1998) and the Keck $10 \mathrm{~m}$ telescope (Vogt et al. 2000).

\section{HD 70642}

HD 70642 (HIP 40952, SAO 199126) is a nearby G5 dwarf, with a distance of $28.8 \mathrm{pc}$ (Perryman et al. 1997), a $V$ magnitude of 7.17, and an absolute magnitude of $M_{V}=4.87$. The star is photometrically stable within the Hipparcos measurement error (0.01 mag). The star is chromospherically inactive, with $\log R^{\prime}(\mathrm{HK})=-4.90 \pm 0.06$, determined from AAT/UCLES spectra of the Ca II H and K lines (Tinney et al. 2002b; C. G. Tinney, C. McCarthy, H. R. A. Jones, R. P. Butler, B. D. Carter, G. W. Marcy, \& A. J. Penny 2003, in preparation). Figure 1 shows the $\mathrm{H}$ line compared with the Sun. The chromospherically inferred age of HD 70642 is $\sim 4$ Gyr.

Spectral synthesis (LTE) of our AAT/UCLES spectrum of HD 70642 yields $T_{\text {eff }}=5670 \pm 20 \mathrm{~K}$ and $V \sin i=2.4 \pm 1$ $\mathrm{km} \mathrm{s}^{-1}$, consistent with its status as a middle-aged G5 dwarf. Like most planet-bearing stars, HD 70642 is metal-rich relative to the Sun. We estimate that $[\mathrm{Fe} / \mathrm{H}]=+0.16 \pm 0.02$ from spectral synthesis, in excellent agreement with the photometric 


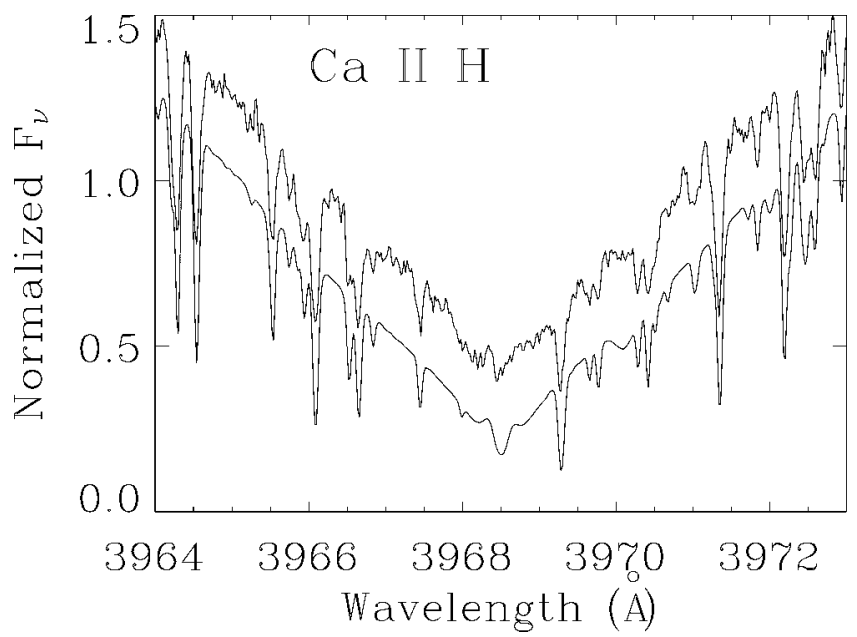

FIG. 1.-Ca II H line of HD 70642 (upper line) compared with the Sun (lower line). HD 70642 is a near-solar twin in both mass and chromospheric activity. Chromospherically quiescent stars such as these are intrinsically stable at the $3 \mathrm{~m} \mathrm{~s}^{-1}$ level.

determination of Eggen (1998). While Ni tracks Fe for most $\mathrm{G}$ and $\mathrm{K}$ dwarfs, the $[\mathrm{Ni} / \mathrm{H}]=+0.22 \pm 0.03$ appears slightly high for HD 70642. The mass of HD 70642 estimated from $B-V, M_{\mathrm{bol}}$, and $[\mathrm{Fe} / \mathrm{H}]$ is $1.0 \pm 0.05 M_{\odot}$.

A total of 21 precision Doppler measurements of HD 70642 spanning more than 5 years are listed in Table 1 and shown in Figure 2. The solid line in Figure 2 is the best-fit Keplerian orbit. The Keplerian parameters are listed in Table 2. The reduced $\chi_{\nu}^{2}$ of the Keplerian fit is 1.4. Figure 3 shows the orbital eccentricity versus the semimajor axis for the extrasolar planets listed by the IAU Working Group on Extrasolar Planets and HD 70642. Solar system planets out to Jupiter are included for comparison. HD 70642b joins 47 UMa c (Fischer et al. 2002) as the only planets yet found in an approximately circular $(e \leq 0.1)$ orbit beyond $3 \mathrm{AU}$.

\section{DISCUSSION}

Prior to the discovery of extrasolar planets, planetary systems were predicted to be architecturally similar to the solar system

TABLE 1

VELOCITIES FOR HD 70642

\begin{tabular}{ccc}
\hline \hline $\begin{array}{c}\text { JD } \\
(-2,450,000)\end{array}$ & $\begin{array}{r}\text { Radial Velocity } \\
\left(\mathrm{m} \mathrm{s}^{-1}\right)\end{array}$ & $\begin{array}{c}\text { Uncertainty } \\
\left(\mathrm{m} \mathrm{s}^{-1}\right)\end{array}$ \\
\hline $830.1082 \ldots \ldots$ & -25.8 & 4.2 \\
$1157.2263 \ldots \ldots$ & -36.4 & 4.4 \\
$1213.1051 \ldots \ldots$ & -42.6 & 4.3 \\
$1236.0850 \ldots \ldots$ & -37.5 & 5.2 \\
$1630.0095 \ldots \ldots$ & -15.9 & 3.6 \\
$1717.8810 \ldots \ldots$ & -9.4 & 3.9 \\
$1920.1348 \ldots \ldots$ & 15.0 & 4.8 \\
$1983.9687 \ldots \ldots$ & 13.7 & 5.8 \\
$2009.0210 \ldots \ldots$ & 13.2 & 4.6 \\
$2060.8744 \ldots \ldots$ & 27.7 & 3.4 \\
$2420.9072 \ldots \ldots$ & 12.7 & 3.1 \\
$2424.8981 \ldots \ldots$ & 11.4 & 3.1 \\
$2455.8416 \ldots \ldots$ & 20.0 & 2.8 \\
$2592.2229 \ldots \ldots$ & 12.7 & 2.9 \\
$2595.2255 \ldots \ldots$ & 15.2 & 3.4 \\
$2710.0700 \ldots \ldots$ & 0.8 & 3.0 \\
$2744.9571 \ldots \ldots$ & 0.3 & 3.1 \\
$2747.9155 \ldots \ldots$ & -4.2 & 2.7 \\
$2749.9755 \ldots \ldots$ & -7.4 & 2.2 \\
$2751.9384 \ldots \ldots$ & -5.6 & 2.4 \\
$2785.9082 \ldots \ldots$ & -3.4 & 2.4 \\
\hline
\end{tabular}

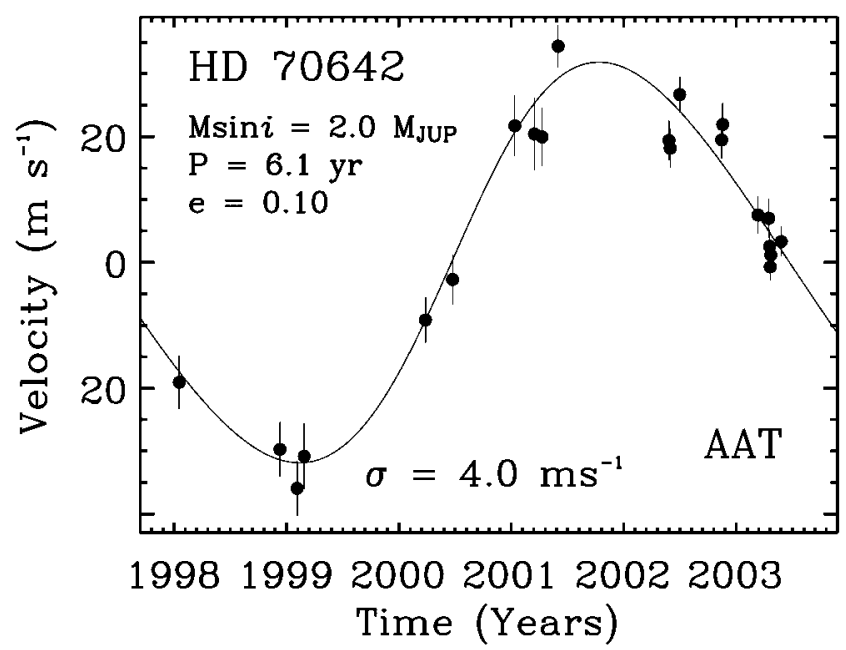

FIG. 2.-Doppler velocities for HD 70642. The best-fit Keplerian orbit is shown as a solid line, with period $P=6.1 \mathrm{yr}$, semiamplitude $K=32 \mathrm{~m} \mathrm{~s}^{-1}$, and eccentricity $e=0.10$, yielding $M \sin i=2.0 M_{\mathrm{JUP}}$. The rms to the Keplerian fit, $4.0 \mathrm{~m} \mathrm{~s}^{-1}$, is consistent with the measurement uncertainty.

(Lissauer 1995; Boss 1995), with giant planets orbiting beyond $4 \mathrm{AU}$ in circular orbits and terrestrial mass planets inhabiting the inner few AU. The landscape revealed by the first $\sim 80$ extrasolar planets is quite different. Extrasolar planetary systems have proved to be much more diverse than imagined, as predicted by Lissauer (1995): "The variety of planets and planetary systems in our Galaxy must be immense and even more difficult to imagine and predict than was the diversity of the outer planet satellites prior to the Voyager mission."

The discovery here of a Jupiter mass planet in a circular orbit highlights the existence, but also the rarity, of giant planets that seem similar to the original theoretical predictions. Review of all the known extrasolar planets, both those described in refereed, published journals (see footnote 8) and those in the larger list of claimed extrasolar planets ${ }^{9}$ shows that $\sim 7 \%$ of extrasolar planets orbiting beyond $0.5 \mathrm{AU}$ reside in circular orbits $(e<0.1)$. Further detections of planets beyond $1 \mathrm{AU}$ are needed to determine if circular orbits are more common for planets that orbit farther from the host star.

Over the next decade, precision Doppler programs will continue to be the primary means of detecting planets orbiting stars within $50 \mathrm{pc}$. By the end of this decade, Doppler programs carried out at precisions of $3 \mathrm{~m} \mathrm{~s}^{-1}$ or better by our group and by others (e.g., Mayor \& Santos 2002) will be sensitive to Jupiter and Saturn mass planets orbiting beyond 4 AU. The central looming question is whether these planets will com-

${ }^{9}$ See http://exoplanets.org.

TABLE 2

Orbital SOLUTION FOR HD 70642

\begin{tabular}{|c|c|c|}
\hline Parameter & Value & Uncertainty \\
\hline Orbital period $P$ (days) & 2231 & 400 \\
\hline Velocity semiamplitude $K\left(\mathrm{~m} \mathrm{~s}^{-1}\right)$ & 32 & 5 \\
\hline Eccentricity $e$ & 0.10 & 0.06 \\
\hline Periastron date (JD) & $2,451,749$ & 300 \\
\hline$\omega(\operatorname{deg}) \ldots \ldots \ldots \ldots$ & 277 & 75 \\
\hline$M \sin i\left(M_{\mathrm{IUP}}\right) \ldots \ldots \ldots \ldots$ & 2.0 & \\
\hline Semimajor axis $(\mathrm{AU}) \ldots \ldots \ldots \ldots \ldots \ldots$ & 3.3 & \\
\hline 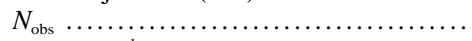 & 21 & \\
\hline $\operatorname{rms}\left(\mathrm{m} \mathrm{s}^{-1}\right)$ & 4.0 & \\
\hline
\end{tabular}




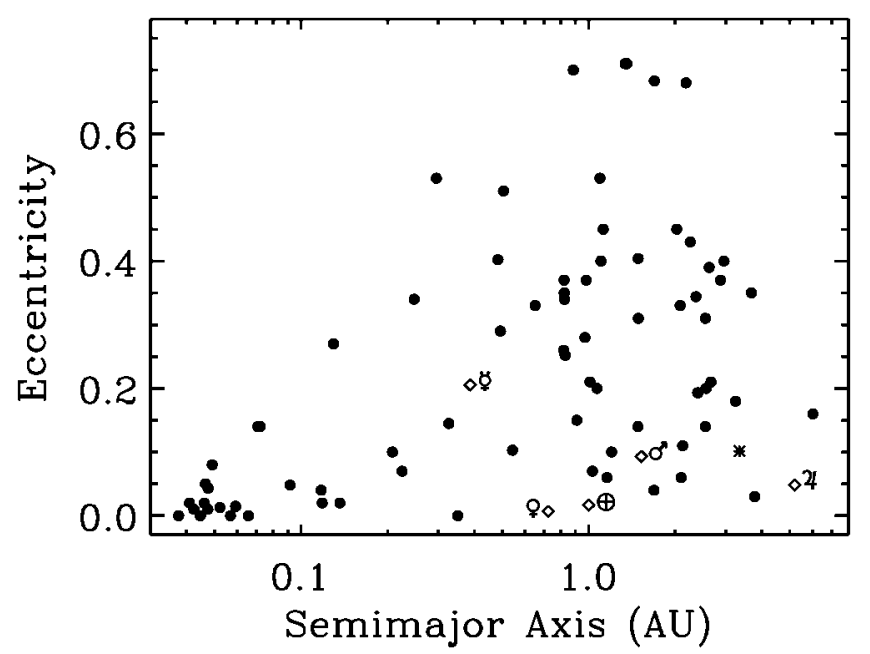

FIG. 3.-Orbital eccentricity vs. semimajor axis for the planet orbiting HD 70642 (asterisk), extrasolar planets listed by the IAU Working Group on Extrasolar Planets (filled circles), and solar system planets out to Jupiter (diamonds with accompanying planetary symbols). HD $70642 \mathrm{~b}$ joins $47 \mathrm{UMa} \mathrm{c}$ as the only planets yet found in an approximately circular orbit $(e \leq 0.1)$ beyond $3 \mathrm{AU}$.

monly be found in circular orbits or whether the architecture of the solar system is rare.

Of the greatest anthropocentric interest are planets in intrinsically circular orbits, as opposed to the short-period planets in tidally circularized orbits. NASA and ESA have made plans for new telescopes to detect terrestrial mass planets. Transit missions such as COROT, Kepler, and Eddington may be sensitive to terrestrial mass planets orbiting near $1 \mathrm{AU}$, providing valuable information on the incidence of such planets. As terrestrial planets make photometric signatures of 1 part in 10,000, these missions may be subject to interpretive difficulties that already challenge current ground-based transit searches for Jupiter-sized planets. Transit missions cannot determine orbital eccentricity and thus cannot determine whether planets are solar system analogs. These space-based transit missions are targeting stars at several hundred parsecs, making follow-up by other techniques difficult.

Ground-based interferometric astrometry programs at Keck and at the Very Large Telescope are projected to begin taking data by the end of this decade. These programs are complementary to existing precision Doppler velocity programs in that they are most sensitive to planets in distant orbits. Like Doppler velocities, astrometry needs to observe one or more complete orbits to make a secure detection and solve for orbital param- eters. It is thus likely that the first significant crop of groundbased astrometry planets will emerge after 2015 .

The NASA Space Interferometry Mission (SIM) is scheduled to launch in 2009. A key objective of the SIM is the detection of planets as small as 3 Earth masses in 1 AU orbits around the nearest stars. SIM offers the promise of determining actual masses of terrestrial planets, thereby securing their status unambiguously. Simulations based on the SIM measurement specifications, along with the proposed target stars, the $5 \mathrm{yr}$ mission lifetime, and a planet mass function extrapolated to the Earth mass regime, ${ }^{10}$ yield predictions that as many as approximately five terrestrial planets could be found (Ford \& Tremaine 2003). Giant planets orbiting 2-5 AU from the host stars are also detectable with SIM, although the orbital parameters will not be well determined in a $5 \mathrm{yr}$ mission. A $10 \mathrm{yr} S I M$ would yield significantly better orbital determinations for Jupiter analogs. Overall, the detection capabilities of SIM are similar to existing precision Doppler programs with a precision of $3 \mathrm{~m} \mathrm{~s}^{-1}$ (Ford \& Tremaine 2003), thereby providing confirmation of known planets and unambiguous masses.

Direct imaging missions such as the NASA Terrestrial Planet Finder $(T P F)$ and the ESA Darwin have the primary goal of detecting Earth-like planets and of obtaining low-resolution spectra that might reveal biomarkers. Such missions will not return dynamical information and hence will not directly reveal the masses of detected planets. Current plans, perhaps optimistically, call for the launching of such missions around 2015. We expect that continued Doppler measurements, as well as future astrometric missions, will contribute significantly to the interpretation of the unresolved companions detected by TPF/Darwin.

We acknowledge support from NSF grant AST 99-88087, NASA grant NAG5-12182, and travel support from the Carnegie Institution of Washington to R. P. B., from NASA grant NAG5-8299 and NSF grant AST 95-20443 to G. W. M., and from Sun Microsystems. We also wish to acknowledge the support of the NASA Astrobiology Institute. We thank the Australian (ATAC) and UK (PATT) Telescope assignment committees for allocations of AAT time. We are grateful for the extraordinary support we have received from the AAT technical staff-E. Penny, R. Paterson, D. Stafford, F. Freeman, S. Lee, J. Pogson, and G. Schaffer. We would especially like to express our gratitude to the AAO Director, Brian Boyle. The AAT Planet Search Program was created and thrived under Brian's tenure as Director in large measure because of his critical support and encouragement. The Australia Telescope National Facility (ATNF) is fortunate to have Brian as their new Director.

${ }^{10}$ A power-law extrapolation admittedly fraught with uncertainty.

\section{REFERENCES}

Boss, A. P. 1995, Science, 267, 360

Butler, R. P., \& Marcy, G. W. 1996, ApJ, 464, L153

1998, in ASP Conf. Ser. 134, Brown Dwarfs and Extrasolar Planets, ed. R. Rebolo, E. L. Martin, \& M. R. Zapatero Osorio (San Francisco: ASP), 162

Butler, R. P., et al. 2002, ApJ, 578, 565

Butler, R. P., Marcy, G. W., Williams, E., McCarthy, C., Dosanjh, P., \& Vogt, S. S. 1996, PASP, 108, 500

Butler, R. P., Tinney, C. G., Marcy, G. W., Jones, H. R. A., Penny, A. J., \& Apps, K. 2001, ApJ, 555, 410

Diego, F., Charalambous, A., Fish, A. C., \& Walker, D. D. 1990, Proc. SPIE, 1235,562

Eggen, O. J. 1998, AJ, 115, 2397

Fischer, D. A., Marcy, G. W., Butler, R.P., Laughlin, G., \& Vogt, S. S. 2002, ApJ, 564, 1028
Ford, E. B., \& Tremaine, S. 2003, PASP, in press

Jones, H. R. A., Butler, R. P., Tinney, C. G., Marcy, G. W., Penny, A. J., McCarthy, C. \& Carter, B. D. 2002a, MNRAS, 337, 1170

. 2003, MNRAS, 341, 948

Jones, H. R. A., Butler, R. P., Tinney, C. G., Marcy, G. W., Penny, A. J., McCarthy, C., Carter, B. D., \& Pourbaix, D. 2002b, MNRAS, 333, 871

Lissauer, J. J. 1995, Icarus, 114, 217

Marcy, G. W., \& Butler, R. P. 1992, PASP, 104, 270

Marcy, G. W., Butler, R. P., Fischer, D. A., Laughlin, G., Vogt, S. S., Henry, G. W., \& Pourbaix, D. 2002, ApJ, 581, 1375

Mayor, M., \& Santos, N. C. 2002, in The Origins of Stars and Planets: The VLT View, ed. J. F. Alves \& M. J. McCaughrean (New York: Springer), 373

Perryman, M. A. C., et al. 1997, A\&A, 323, L49 
Tinney, C. G., Butler, R. P., Marcy, G. W., Jones, H. R. A., Penny, A. J., McCarthy, C., \& Carter, B. D. 2002a, ApJ, 571, 528

Tinney, C. G., Butler, R. P., Marcy, G. W., Jones, H. R. A., Penny, A. J., McCarthy, C., Carter, B. D., \& Bond, J. 2003, ApJ, 587, 423

Tinney, C. G., Butler, R. P., Marcy, G. W., Jones, H. R. A., Penny, A. J., Vogt, S. S., \& Henry, G. W. 2001, ApJ, 551, 507
Tinney, C. G., McCarthy, C., Jones, H. R. A., Butler, R. P., Carter, B. D., Marcy, G. W., \& Penny, A. J. 2002b, MNRAS, 332, 759

Valenti, J. A., Butler, R. P., \& Marcy, G. W. 1995, PASP, 107, 966

Vogt, S. S., Butler, R. P., Marcy, G. W., Fischer, D. A., Pourbaix, D., Apps, K., \& Laughlin, G. 2002, ApJ, 568, 352

Vogt, S. S., Marcy, G. W., Butler, R. P. \& Apps, K. 2000, ApJ, 536, 902 